\title{
INTERVISTA A NANNI BALESTRINI
}

José Molina*

In questa intervista il poeta Nanni Balestrini spiega il modo aleatorio da lui utilizzato nel 1961 per comporre il suo poema "Tape Mark", che é stato uno dei primi esempi di poesia fatta col computer. Balestrini ha continuato a lavora con la composizione aleatoria anche per il romanzo "Tristano" e per il film "Tristan Oil".

\section{T. D .: Nanni ci siamo visti a Milano a luglio quest'anno e mi raccontavi sulla possibilità combinatoria del computer in uno dei tuoi libri, il "Tristano".}

N. Balestrini: Prima io avevo fatto poesia combinatoria, elettronica con tape-mark nel 61. Poi avevo in mente di fare ancora una cosa più ampia con la prosa e avevo fatto un testo con frasi e collage di frasi diverse, di vario tipo che si potevano mescolare tra loro e combinare diversamente. E questo libro: il "Tristano", I'ho pubblicato nel 1966, credo, però la mia idea era di fare una cosa combinatoria nel senso che si potevano ricombinare ogni volta i brani di questo testo e fare delle copie diverse. Però allora non si poteva fare questo. L'ho fatto adesso perché c'è la stampa digitale perché con la stampa normale, con la stampa tipografica, tutte le copie sono uguali. Quando la stampa è diventata digitale dieci anni fa, questo libro è uscito nel 2007, ho potuto stamparlo con queste macchine digitali, che si usano per fare delle piccole tirature, con quelle si possono stampare una cinquanta di copie o le copie che vuoi. Come procedimento io ho fatto creare col computer 2000 diverse combinazioni, la combinazione è una cosa molto semplice, c'è una serie di capitoli composti per venti

\footnotetext{
" PhD Candidate - Erasmus Mundus Joint Doctorate "Cultural Studies in Literary Interzones" (Università di Tubingen, Università di Barcellona, Università di Perpinyan). Intervista realizzata durante il semestre di studi presso il gruppo di ricerca Hermeneia, direzione nazionale dell' Erasmus Mundus Joint Doctorate.
}

Esta obra está licenciada com uma licença Creative Commons. 
brani e per cui io ho dato semplicemente due regole: una è quella di variare questi venti brani all'interno di un capitolo, prima io avevo scritto trenta e così si potevano scegliere soltanto venti. La seconda è sceglierne venti e metterli n'un ordine casuale. La seconda istruzione è quella di cambiare l'ordine dei capitoli. E con queste due regole vengono fuori un numero di possibili combinazioni che sono miliardi. Qui c'è anche la prefazione di Umberto Eco, che spiega anche un po' questo. Per cui io ho stampato queste copie, in principio duemila e dopo altre cinquecento. Poi è uscita l'edizione tedesca ed anche loro hanno fatto due o tre mila copie ed si vede in copertina il loro numero. Poi è uscita quella inglese e anche la francese.

\section{T.D.: Manca la spagnola.}

N. Balestrini: Sì, manca quella. lo l'avevo anche proposta al direttore di "Anagrama" a Barcellona ma mi ha detto che era troppo difficile.

\section{T.D.: Quali testi hai pubblicato a Barcellona?}

N. Balestrini: Ho pubblicato "Invisibili" ed a Madrid c'è una piccola casa editrice, "Traficantes del sueño", politicamente di sinistra, che ha stampato tutti i miei libri politici ma quella non è adatta per fare una cosa col Tristano.

\section{T. D.: Cosa ci vuole per fare un'edizione di questo libro?}

N. Balestrini: Col computer, è stato un tecnico informatico che mi ha fatto questa cosa, si prendono tutti questi brani e si ricombinano al computer, fa due mila combinazioni, in ogni combinazione c'è tutto il libro. Ogni volta ricombinata in un modo diverso come ti ho detto. E così abbiamo due mila libri in digitale, lui ha messo tutto questo su un disco e si stampa come se fosse un libro solo poi viene diviso e vengono fatte le copertine, ognuna col suo numero diverso. I traduttori al francese e 
tedesco hanno fatto le traduzioni e poi le hanno spedite a questo mio amico tecnico. Anzi l'edizione inglese è stata stampata qui in Italia con la stessa tipografia.

Tutte queste edizioni sono diverse, non solo nel senso che lo stesso materiale è combinato in un ordine diverso ma anche, visto che, come ti ho detto, sono scelti per ogni capitolo venti brani su trenta, così ci sono delle cose che in un altro libro non ci sono . Ed allora a me interessava prima di tutto l'idea del libro personale, una copia di questo ce l'ha solo quello che ha comprato questa copia, per cui è una storia sua, una storia personale. Ma poi anche perché la mia idea è che questo è più vicino $a$ un fatto biologico, naturale. Perché la stampa meccanica, come tutta la meccanica, fa degli oggetti che sono tutti uguali. Mentre, invece, la produzione della natura fa tutte le cose diverse come gli alberi o gli uomini, siamo sempre un po' diversi e così il mio libro voleva essere un po' biologico.

Questo principio della stampa digitale si applica oggidi all'industria con un programma che personalizza le caratteristiche dell'oggetto, le distribuzioni degli attrezzi, etc. A me m'affascinava questa idea di uscire dalla meccanica perché la meccanica è una gabbia che ti fa tutto uguale.

\section{T.D.: Poi quando hai fatto questo libro già avevi un rapporto col computer?}

N. Balestrini: Si c'era già il computer perché prima quando ho fatto quelle cose negli anni sessanta non c'era come oggi. C'erano i calcolatori che erano questi grandi armadi con le luci che si accendevano ma certamente tutto questo è cambiato completamente. Però non bisogna neanche pensare che ci siano tante cose che si possono fare. Io non sono un fanatico del computer e dell'elettronica, si possono realizzare alcune idee che tu hai ma non inventano niente. $\dot{E}$ uno strumento che fa tutto quello che tu gli dici di fare, il grande vantaggio è che lo fa più in fretta, con una grande rapidità. Se io tentasse di fare il Tristano a mano mi prenderebbe alcuni anni. 


\section{T. D.: Tu sei uno dei primi poeti italiani ad avere esplorato il computer nella poesia.}

N. Balestrini: Ma questo qui io non lo so bene perché c'erano stati certamente altre cose. Però i critici in Germania ed Inghilterra dicono che io sono stato il primo a fare una poesia con il calcolatore elettronico. Non lo so, a un certo punto ho provato una cosa molto semplice, le due cose per cui mi interessava fare questa prima poesia erano: una, la rapidità delle combinazioni. Nella poesia è più semplice che nella narrativa perché i poeti hanno fatto sempre, oltre che la poesia è così, un lavoro combinatorio. Una forma come la sestina è una summa delle combinazioni. Le rime, le strofe, quella è naturalmente combinatoria. Anche un poeta quando scrive le poesie, prende le parole e le sposta,è tutto un fatto di organizzazione. Ora tutto questo, se tu dai delle istruzioni il computer le esegue e le fa con una rapidità straordinaria. L'altra cosa interessante di questo è che in queste combinazioni il caso ti da delle opzioni che tu non potevi prevedere. Anche tu puoi fare le cose casuali, mescoli le parole come facevano i surrealisti, mettendo le parole in pezzi di carta in un cappello e poi le tiravano fuori come vengono e così facevano una poesia.

\section{T. D.: Sei soddisfatto con i risultati di quel primo lavoro?}

N. Balestrini: Sì, sì. Ma fatto una volta non è che tutti i libri li farei così neppure quello delle copie diverse, ne fai uno e basta. Fu un esempio, una prova ma da farsi una volta sola. Poi da questo ho fatto un film che si chiama "Tristan Oil", con lo stesso principio combinatorio. II titolo corrisponde al mio romanzo "Tristano" e "oil" perché l'ho fatto su i danni ecologici che produce il petrolio. La distruzione del pianeta attraverso lo sfruttamento delle risorse naturali, specialmente del petrolio. Qui invece di pigliare dei pezzi di scrittura, ho preso dei pezzi di video, di documentari video, che sono, per la maggior parte, fatti di questi fenomeni d'inquinamento e poi anche delle cose legate al petrolio come Wall Street, la borsa, le finanze. Dopo ho messo dei pezzi di un vecchia serie televisiva che si chiamava "Dallas", perché quelli erano dei 
petrolieri. II meccanismo è lo stesso di questo, ho scelto un grande magazzino di questi piccoli frammenti di trenta secondi o un minuto massimo e poi ho dato un'istruzione al computer di combinarli fra di loro casualmente in sequenze in pezzi di dieci minuti. Ogni dieci minuti questo film pesca un ordine casuale e li mette insieme, poi li pesca un altro e tutto questo è un po' sovrapposto ad una specie di flusso di petrolio. Allora questo è come il libro, il libro è, praticamente, il numero di copie che si possono fare ed è infinito perché matematicamente si arriva a un numero finito, forse tre miliardi, ma questo è un po' come il lotto dove capita che a volte i numeri si ripetono. E così questo film gira giorno e notte continuamente e ho fatto cinquemila dvd diversi, con le gallerie e musei italiani di una decina di città, l'ultima è stata Venezia. Adesso continua ad andare a Napoli nel museo Madre. Questo l'ho fatto due anni fa, nel 2012.

\section{T. D.: Mi raccontavi dei Novissimi e di come Edoardo Sanguinetti aveva dato il nome.}

N. Balestrini: Novissimi è stato nella mia generazione in un periodo di trasformazione della Italia. C'è stata la guerra e il dopoguerra alla fine degli anni cinquanta e primi anni sessanta. L' Italia si è trasformata in poco tempo in un paese industriale chiamato il miracolo economico perché da essere un paese sostanzialmente agricolo in due o tre anni è diventato moderno ed industriale. Tutto questo aveva portato un grande cambiamento e soprattutto con gli scrittori, tutti quelli che lavoravano con la lingua perché allora la lingua italiana era poco usata, tutti parlavano in dialetto, ognuno aveva il suo dialetto che assomigliava un po'al l'italiano. Il cambiamento è stato con la migrazione degli operai cha venivano dal sud a lavorare nelle fabbriche del nord e ci voleva la lingua per capirsi tra di loro.

Poi l'altro fenomeno che ha diffuso la lingua è stata la televisione che aveva cominciato a trasmettere a metà degli anni cinquanta. $\mathrm{E}$ il terzo fattore è stato la scuola dell'obbligo, una legge che diceva che tutti dovevano frequentare la scuola 
fino a quattordici anni. L'italiano moderno non è quello di Dante, il fiorentino, perché quello che si parla oggi ha una influenza dal romano perché la televisione e il cinema erano fatti a Roma.

Dunque, questa era la lingua nella quale noi dovevamo scrivere. Anche la grande scoperta degli autori europei come Joyce e Kafka ha propiziato la fondazione di una nuova letteratura italiana promossa con gli autori del gruppo 63 , la generazione degli anni sessanta.

I Novissimi è stata una antologia di cinque poeti italiani pubblicata nel sessantuno. Una poesia che voleva esser di rottura e di avanguardia scritta con la nuova lingua italiana parlata. I poeti pubblicati erano Edoardo Sanguinetti, Elio Pagliarini, Antonio Porta, Alfredo Giuliani ed io, noi tutti siamo entrati anche nel gruppo 63. Nel gruppo 63 non si parlava soltanto di poesia, erano riunioni dove entrava anche la narrativa e la critica, una idea presa dal gruppo 47 in Germania. In quel gruppo ogni scrittore leggeva un brano del suo lavoro e tutti gli altri criticavano e dicevano cosa ne pensavano. Così con quel giudizio è venuta fuori una letteratura splendida come Gunther Grass, Enzenberger, Bachman, etc.

\section{ENTREVISTA COM NANNI BALESTRINI}

RESUMO: Nesta entrevista, o poeta italiano Nanni Balestrini explica a sua escolha do método aleatório de composição na elaboração do seu poema "Tape Mark" feito em 1961, o qual tem sido uma pedra de toque para a composição da poesia no computador. O poeta tem utilizado este procedimento também na elaboração do seu romance "Tristano" e do filme "Tristan Oil".

\section{INTERVIEW WITH NANNI BALESTRINI}

Texto Digital, Florianópolis, Santa Catarina, Brasil, v. 11, n. 2, p. 185-191, jul./dez. 2015. ISSNe: 1807-9288. 
ABSTRACT: In this interview, the Italian poet Nanni Balestrini explains the aleatoric procedure he first used in 1961 with his master piece "Tape Mark", one of the first precedents and milestone of the computer composition in poetry. A similar procedure is used in the composition of his romance "Tristano" and of a short film "Tristan Oil".

Texto Digital, Florianópolis, Santa Catarina, Brasil, v. 11, n. 2, p. 185-191, jul./dez. 2015. ISSNe: 1807-9288. 